\title{
Modeling the strings in the outer regions of $\eta$ Carinae
}

\author{
Matt P. Redman ${ }^{1}$, John Meaburn ${ }^{2}$, and Anthony J. Holloway ${ }^{2}$ \\ ${ }^{1}$ Department of Physics and Astronomy, University College London, \\ Gower Street, London WC1E 6BT, UK \\ ${ }^{2}$ Jodrell Bank Observatory, University of Manchester, \\ Macclesfiled SK11 9DL, UK
}

\begin{abstract}
The narrow high-speed optical filaments emerging from the Homunculus of $\eta$ Carinae are modelled as resulting from the passage of ballistic 'bullets' of material through the dense circumstellar environment. In this explanation, the string is the decelerating flow of ablated gas from the bullet. Many of the observed properties of the strings are accounted for in this simple model.
\end{abstract}

\section{Observations and explanations}

Several high speed, narrow filaments of optically emitting gas are observed to protrude radially from the Homunculus of $\eta$ Car and are referred to as 'spikes', 'whiskers' or 'strings' (e.g., Meaburn, Wolstencroft \& Walsh 1987; Weis, Duschl \& Chu 1999); Weis et al. (1999), have investigated their peculiar properties. They are highly collimated, but have localised kinks and brightness knots. The velocity increases linearly along the length of the string from about $-630 \mathrm{~km} \mathrm{~s}^{-1}$ at the base to up to about $-850 \mathrm{~km} \mathrm{~s}^{-1}$ at the tip. All portions of the string have the same kinematical age as the Homunculus. The HST image in Figure 1 is of the brightest and most easily observed string - see Weis et al. (1999) for images of other strings. Currie et al. (2000) discovered that the unresolved point source labelled in Figure 1 has a velocity consistent with the linear increase along the string. This implies the knot and string are closely related.

Several possible explanations for the origin of the strings can be considered (Weis et al. 1999; Soker 2001; Redman, Meaburn \& Holloway 2002). A collimated jet or edge brightened funnel is unlikely, because of the number of strings (at least five). Beams of radiation, escaping through gaps in Homunculus and scattering off dust, can be ruled out, since the spikes are kinked rather than perfectly straight. Ionization shadows behind dense clumps, resulting in columns of gas that are ionized later than the surrounding medium, do not account for the velocity structure and dense downstream knot. Trains of individual bullets following the same path would require the bullets to have been ejected in the same direction at the same time, but with a range of speeds.

In Redman et al. (2002), we suggest that the strings are simply the effects of the passage of dense fragments of ejecta interacting with the circumstellar environment. The visible string is the cooling trail left behind by the passage of the ejected fragment, as it passes through the circumstellar environment. The bullets are assumed to have been generated in the 1840s outburst that created 


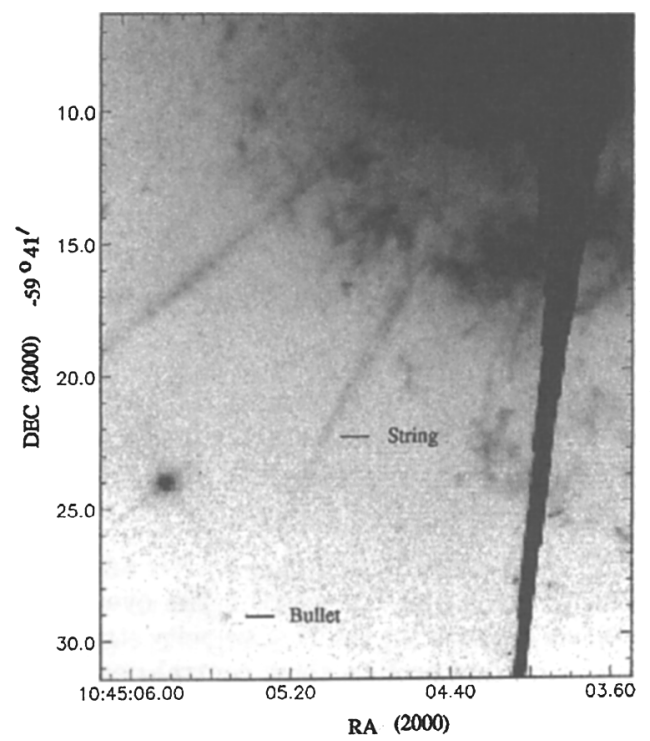

Figure 1. A HST archive image of $\eta$ Car String 1 in the light of $[\mathrm{N}$ II] $\lambda 6584 \AA$.

the Homunculus. The string will have only expanded by $c_{\mathrm{i}} t_{\mathrm{dyn}} \lesssim 4.5 \times 10^{15} \mathrm{~cm}$ in the $150 \mathrm{yr}$ since the outburst. This is less than the current thickness of the strings and the clump must therefore also have had an initial diameter less than that of the string. The clump will be destroyed before it has swept up a column of material equal to itself, i.e., in a time $t_{\text {dest }}=\chi r_{\mathrm{c}} / V_{\mathrm{s}}$, where $\chi$ is the density contrast between the clump and the medium. Since the clump still survives after 150 years, it must have a density of around 100 times that of the local environment - a modest requirement. Localised kinks and bright knots in the string could be caused by the Kelvin-Helmholtz instability, as the string gas mixes with the ambient gas. The displacement between the bullet and the string (see Figure 1) is likely due to the newly shocked material, stripped from the clump, having to cool before becoming optically visible. The cooling timescale required is consistent with that expected from gas of $\sim 10^{5} \mathrm{~K}$ cooling to $\sim 10^{4} \mathrm{~K}$. Meanwhile, the clump emission is due to a slow shock, being driven into the dense clump. The decreasing velocity along the string could be due to the gradual slowing of the string gas, as it mixes with the slower moving circumstellar medium. The older parts of the string, closest to $\eta \mathrm{Car}$, will have been slowed more than those parts more recently stripped from the bullet surface.

\section{References}

Currie, D., Le Mignant, D., Svensson, B., et al. 2000, ESO Messenger 102, 25

Meaburn, J., Wolstencroft, R.D., Walsh J.R. 1987, A\&A 181, 333

Redman, M. P., Meaburn, J., Holloway, A.J. 2002, MNRAS 332, 754

Soker, N. 2001, ApJ 377, 672

Weis, K., Duschl, W.J., Chu Y.-H. 1999, A\&A 349, 467 